Volume 133, Number 9, Pages 2705-2710

S 0002-9939(05)07817-2

Article electronically published on March 22, 2005

\title{
ELLIPSES, NEAR ELLIPSES, AND HARMONIC MÖBIUS TRANSFORMATIONS
}

\author{
MARTIN CHUAQUI, PETER DUREN, AND BRAD OSGOOD
}

(Communicated by Juha M. Heinonen)

\begin{abstract}
It is shown that an analytic function taking circles to ellipses must be a Möbius transformation. It then follows that a harmonic mapping taking circles to ellipses is a harmonic Möbius transformation.
\end{abstract}

Analytic Möbius transformations take circles to circles. This is their most basic, most celebrated geometric property. We add the adjective 'analytic' because in a previous paper [1] we introduced harmonic Möbius transformations as a generalization of Möbius transformations to harmonic mappings. Their basic geometric property, the only one we know so far, is that they take circles to ellipses. In this paper we consider the converse question. We shall show that a harmonic mapping that takes circles to ellipses must be a harmonic Möbius transformation. We also have some comments on the situation for analytic functions; in fact, we need a similar result for analytic functions to deal with the harmonic case.

\section{HARMONiC MAPPINGS AND haRMONiC MÖBIUS TRANSFORMations}

We begin with a very brief review of the definition and properties of harmonic mappings and harmonic Möbius transformations, followed by a statement of our main result. A harmonic, complex-valued function $f$ defined on a simply connected domain can be written in the form $f=h+\bar{g}$, where $h$ and $g$ are analytic. When $f$ is locally univalent and sense-preserving one has $h^{\prime}(z) \neq 0$ and the analytic function $\omega=g^{\prime} / h^{\prime}$, called the (second) complex dilatation of $f$, satisfies $|\omega(z)|<1$. In this paper we will always assume that a harmonic function $f$ is locally univalent and sense-preserving, and we refer to $f$ as a harmonic mapping.

On any neighborhood where $\omega$ is not zero or has zeros of even order, $f$ lifts to a mapping whose image is a minimal surface in $\mathbb{R}^{3}$. The metric of the surface has the form $\rho|d z|$ where $\rho=\left|h^{\prime}\right|+\left|g^{\prime}\right|$ and the curvature is

$$
K=-\frac{\left|\omega^{\prime}\right|^{2}}{\left|h^{\prime}\right|\left|g^{\prime}\right|\left(1+|\omega|^{2}\right)} .
$$

We refer to [2] for further background.

Received by the editors January 22, 2004 and, in revised form, April 29, 2004.

2000 Mathematics Subject Classification. Primary 30C99; Secondary 31A05.

Key words and phrases. Harmonic mapping, Schwarzian derivative, harmonic Möbius transformation, circles, ellipses.

The first author was supported by Fondecyt Grant \# 1030589. 
We need a few facts on the curvature and the dilatation; see [1] for the details. First, the curvature is constant if and only if it is zero. This in turn is equivalent to the dilatation being constant (not necessarily zero), and then to $f$ being of the form $f=h+\alpha \bar{h}$ for some analytic, locally univalent function $h$ and a constant $\alpha$, $|\alpha|<1$.

In [1] we introduced the Schwarzian derivative of a harmonic mapping by the formula

$$
S(f)=2\left[(\log \rho)_{z z}-\left((\log \rho)_{z}\right)^{2}\right]
$$

and developed several properties analogous to those of the Schwarzian of an analytic function. In particular, $S(f)=0$ if and only if $f$ is of the form $f=h+\alpha \bar{h}$ where $\alpha$ is a complex constant with $|\alpha|<1$ and $h$ is an (analytic) Möbius transformation. Pursuing the analogy, we defined a harmonic Möbius transformation to be a harmonic mapping of this form. Since such a map is the composition of an analytic Möbius transformation with the linear map $z \mapsto z+\alpha \bar{z}$, it follows that harmonic Möbius transformations take circles to ellipses. (We also see that a harmonic Möbius transformation is univalent and has a univalent extension to $\mathbb{C}$.) Our purpose here is to close the loop of equivalences.

Theorem. For a harmonic mapping $f$ the following are equivalent:

(i) The Schwarzian derivative $S(f)=0$.

(ii) $f=h+\alpha \bar{h}$ for some (analytic) Möbius transformation $h$ and some complex constant $\alpha$ with $|\alpha|<1$.

(iii) $f$ takes circles to ellipses.

We note that the Radó-Kneser-Choquet theorem (see [2]) implies that there are harmonic mappings of the disk onto a domain bounded by an ellipse, with prescribed boundary correspondence. Because of this flexibility at the boundary such a mapping need not be a harmonic Möbius transformation, and thus one certainly needs more than one circle going to one ellipse to characterize Möbius transformations, harmonic or analytic. The theorem is stated in terms of all circles going to ellipses, and though our proof uses this it may be that one can do with less; see the remarks after the proof of Proposition 1, below.

\section{AnAlytic FUnCtions tAKING CIRCLES TO ELLIPSES}

The first step in proving the theorem is a result for analytic functions.

Proposition 1. An analytic function $f$ on a domain $\Omega$ taking circles to ellipses is a Möbius transformation.

So, it turns out, the mapping is univalent and the image ellipses are actually circles. This result would not surprise anyone, but the proof might. Afterward we discuss an example that was suggested by an alternate argument.

Proof. It must be that $f$ is not constant; thus, as our arguments will be local, we may assume that $f^{\prime} \neq 0$ on $\Omega$ and, by further restricting the domain, that $f$ is univalent. Normalize in the domain and range so that the closed unit disk lies in $\Omega$ and so that $f$ maps $|z|=1$ onto the ellipse

$$
\frac{u^{2}}{a^{2}}+\frac{v^{2}}{b^{2}}=1 \quad \text { with } \quad f(0)=0, f^{\prime}(0)=1 .
$$


Then $f$ is odd and real on the real axis. For $r \leq 1$ the image of the circles $|z-r / 2|=r / 2$ are ellipses symmetric in the real axis, and thus the curvatures at $f(0)$ and $f(r)$ are equal. They are given, respectively, by

$$
\frac{1}{\left|f^{\prime}(0)\right|}\left(\frac{2}{r}+\operatorname{Im}\left\{-i \frac{f^{\prime \prime}(0)}{f^{\prime}(0)}\right\}\right) \text { and } \frac{1}{\left|f^{\prime}(r)\right|}\left(\frac{2}{r}+\operatorname{Im}\left\{i \frac{f^{\prime \prime}(r)}{f^{\prime}(r)}\right\}\right)
$$

so that

because of the normalizations.

$$
\frac{2}{r}=\frac{1}{f^{\prime}(r)}\left(\frac{2}{r}+\frac{f^{\prime \prime}(r)}{f^{\prime}(r)}\right)
$$

A simple integration leads to

$$
f^{\prime}(r)=\frac{1}{1-c r^{2}}, \quad c \in \mathbb{R}, 0<r<1
$$

hence

$$
f^{\prime}(z)=\frac{1}{1-c z^{2}}
$$

for all $z \in \Omega$. From here it would seem clear that we must have $c=0$, whence $f(z)=z$, but it requires some effort to show this. Here is one approach, a direct one, that exhibits some unexpected cancelations.

Let $f=u+i v$. By assumption, the image of $|z|=r$ under $f$ is an ellipse for any $0<r \leq 1$, and the equation is

$$
\frac{u\left(r e^{i \theta}\right)^{2}}{f(r)^{2}}+\frac{v\left(r e^{i \theta}\right)^{2}}{|f(i r)|^{2}}=1
$$

Using

$$
f(z)=z+\frac{c}{3} z^{3}+\frac{c^{2}}{5} z^{5}+\cdots
$$

from the formula for $f^{\prime}(z)$, we have

$$
\begin{aligned}
u\left(r e^{i \theta}\right) & =r\left(\cos \theta+\frac{c}{3} r^{2} \cos 3 \theta+\frac{c^{2}}{5} r^{4} \cos 5 \theta+O\left(r^{6}\right)\right), \\
v\left(r e^{i \theta}\right) & =r\left(\sin \theta+\frac{c}{3} r^{2} \sin 3 \theta+\frac{c^{2}}{5} r^{4} \sin 5 \theta+O\left(r^{6}\right)\right), \\
f(r) & =r\left(1+\frac{c}{3} r^{2}+\frac{c^{2}}{5} r^{4}+O\left(r^{6}\right)\right), \\
|f(i r)| & =r\left(1-\frac{c}{3} r^{2}+\frac{c^{2}}{5} r^{4}+O\left(r^{6}\right)\right) .
\end{aligned}
$$

Substitute into (1) to obtain

$$
\begin{aligned}
(1 & \left.-\frac{c}{3} r^{2}+\frac{c^{2}}{5} r^{4}+O\left(r^{6}\right)\right)^{2}\left(\cos \theta+\frac{c}{3} r^{2} \cos 3 \theta+\frac{c^{2}}{5} r^{4} \cos 5 \theta+O\left(r^{6}\right)\right)^{2} \\
& +\left(1+\frac{c}{3} r^{2}+\frac{c^{2}}{5} r^{4}+O\left(r^{6}\right)\right)^{2}\left(\sin \theta+\frac{c}{3} r^{2} \sin 3 \theta+\frac{c^{2}}{5} r^{4} \sin 5 \theta+O\left(r^{6}\right)\right)^{2} \\
& =\left(1+\frac{c}{3} r^{2}+\frac{c^{2}}{5} r^{4}+O\left(r^{6}\right)\right)^{2}\left(1-\frac{c}{3} r^{2}+\frac{c^{2}}{5} r^{4}+O\left(r^{6}\right)\right)^{2} .
\end{aligned}
$$

Now expand and collect terms. On the left-hand side the constant term is 1 and the coefficient of $r^{2}$ is

$$
\frac{2 c}{3}\left(\cos \theta \cos 3 \theta-\cos ^{2} \theta+\sin \theta \sin 3 \theta+\sin ^{2} \theta\right),
$$


which collapses to 0 . The coefficient of $r^{4}$ is

$$
\begin{gathered}
c^{2}\left\{\left(\frac{1}{9}+\frac{2}{5}\right)\left(\cos ^{2} \theta+\sin ^{2} \theta\right)+\frac{1}{9}\left(\cos ^{2} 3 \theta+\sin ^{2} 3 \theta\right)+\frac{4}{9}(\sin \theta \sin 3 \theta-\cos \theta \cos 3 \theta)\right. \\
\left.+\frac{2}{5}(\cos \theta \cos 5 \theta+\sin \theta \sin 5 \theta)\right\}
\end{gathered}
$$

which simplifies to

$$
\frac{2 c^{2}}{45}(14-\cos 4 \theta)
$$

so the left-hand side of (2) in total is

$$
1+\frac{2 c^{2}}{45}(14-\cos 4 \theta) r^{4}+O\left(r^{6}\right) .
$$

On the other hand, the right-hand side of (2) is

$$
1+\frac{26 c^{2}}{45} r^{4}+O\left(r^{6}\right)
$$

By comparison of the two results it follows that $c=0$, as claimed. This proves Proposition 1.

Another approach to proving this proposition, which we will not present, led us to consider functions of the form

$$
f(z)=z+a_{3} z^{3}+a_{5} z^{5}+\cdots, \quad a_{3} \neq 0,
$$

which approximately map concentric circles $|z|=r$ to concentric ellipses with as small an error as possible. By rescaling $f$ in the domain and range one can assume that $a_{3}=1$. We found that the function

$$
f(z)=z+z^{3}+2 z^{5}+5 z^{7}
$$

satisfies the ellipse equation (1) with an error of size $O\left(r^{8}\right)$. (Of course, when $r$ is small the concentric ellipses are nearly concentric circles.) Furthermore, this is as far as one can go; i.e., no additional term $c z^{9}$ will make $f$ satisfy (1) with an error of order $O\left(r^{10}\right)$.

As mentioned at the beginning of the proof, Proposition 1 is really a local statement, more accurately an infinitesimal statement — for the proof one needs a shrinking sequence of circles mapping to ellipses. We do not know what a more global statement might be. Obviously the Riemann mapping of the unit disk onto the region bounded by an ellipse is not a Möbius transformation, so one circle can map onto one ellipse without any further conclusions being drawn. (This mapping is given in terms of elliptic functions, and, if by some chance you have wondered, we now know for certain that it does not map concentric circles to concentric ellipses.) Though it is true that a multiply connected domain bounded by circles can be mapped conformally onto one bounded by ellipses, we might conjecture that a conformal mapping of a simply connected domain cannot take two circles onto two ellipses. 


\section{HARMONIC MAPPINGS TAKING CIRCLES TO ELLIPSES}

We return now to harmonic mappings, and corresponding to Proposition 1 we have the following local property.

Proposition 2. A harmonic mapping $f$ on a domain $\Omega$ taking circles to ellipses has constant dilatation.

Proof. We will show that the dilatation is constant by showing that the curvature of the minimal surface corresponding to $f$ is zero; recall the remark on this in Section 1. Note that $f$ has no lift only at the discrete set of points that are odd-order zeros of the dilatation, and we may thus confine our analysis to an arbitrary (small) simply connected domain $V$ where the lift exists.

Write $f=h+\bar{g}$ and take any $z_{0} \in V$. By shifting we may assume for simplicity that $z_{0}=0$. We may assume also that $h(0)=g(0)=0$ and that $h^{\prime}(0)=1$. In terms of power series,

$$
h(z)=z+a_{2} z^{2}+\ldots, \quad g(z)=\bar{\alpha} z+\ldots,
$$

and $|\alpha|<1$ because $f$ is sense-preserving. Now, perhaps on a smaller neighborhood $U$ of 0 , consider the harmonic mapping

$$
F(z)=f\left(\frac{z}{1+a_{2} z}\right) .
$$

Note that $F$ still maps circles to ellipses. Analytically, the effect is to make

$$
F(z)=z+\alpha \bar{z}+b_{2} \bar{z}^{2}+O\left(z^{3}\right) ;
$$

i.e., if we write $F=H+\bar{G}$, then $H^{\prime \prime}(0)=0$. Geometrically, $w=z /\left(1+a_{2} z\right)$ is an analytic reparametrization of part of the surface corresponding to $f$. In particular, the curvature is invariant (this is also easy to check directly from the formula for $K)$, and since 0 maps to 0 we have, in obvious notation, $K_{f}(0)=K_{F}(0)$. We will show that $K_{F}(0)=0$.

We claim first that $b_{2}=0$. Suppose by way of contradiction that $b_{2} \neq 0$ and consider the image

$$
\frac{1}{r} F\left(r e^{i \theta}\right)=e^{i \theta}+\alpha e^{-i \theta}+b_{2} r e^{-2 i \theta}+O\left(r^{2}\right)
$$

of $|z|=r$ for small $r$, and the curve

$$
\varphi_{r}(\theta)=e^{i \theta}+\alpha e^{-i \theta}+b_{2} r e^{-2 i \theta} .
$$

The linear part

$$
\psi(\theta)=e^{i \theta}+\alpha e^{-i \theta}
$$

parametrizes an ellipse. Simple considerations show that $\varphi_{r}(\theta)=\psi(\theta)+b_{2} r e^{-2 i \theta}$ has exactly 6 intersections with $\psi(\theta)$ and that the intersections are transverse. $(\psi(\theta)$ goes around once counterclockwise while $b_{2} r e^{-2 i \theta}$ goes around twice clockwise. Incidentally, one can get quite interesting curves by this kind of perturbation of an ellipse.) Adding a term of size $O\left(r^{2}\right)$ will not change the number of intersections; that is, the ellipse (by assumption)

$$
\frac{1}{r} F\left(r e^{i \theta}\right)=\varphi_{r}(\theta)+O\left(r^{2}\right)
$$

intersects the ellipse $\psi(\theta)$ at 6 points. This cannot be, and we conclude that $b_{2}=0$. 
Finally, the curvature of the surface corresponding to the lift of $F$ (and of $f$ ) is

$$
K=-\frac{\left|\omega^{\prime}\right|^{2}}{\left|H^{\prime}\right|\left|G^{\prime}\right|\left(1+|\omega|^{2}\right)},
$$

where $\omega=G^{\prime} / H^{\prime}$. Here we may assume that $G^{\prime} \neq 0$ on the neighborhood (or on a smaller neighborhood), for if $G^{\prime}$ vanishes identically, then $G$ is constant and $F$, hence $f$, is analytic, and the dilatation is zero. The computations with power series now easily imply that $\omega^{\prime}(0)=0$, so that

$$
K(z)=|z|^{2}+\cdots ;
$$

thus $K$ actually vanishes to second order at 0 . Since 0 represents an arbitrary point $z_{0} \in V$, the curvature therefore vanishes identically and the dilatation of $f$ is therefore constant.

\section{Proof of the theorem}

We remarked earlier that the statements (i) and (ii) are equivalent and that (ii) implies (iii). We will complete the proof by showing that (iii) implies (ii).

If a harmonic mapping $f$ takes circles to ellipses, then by Proposition 2 its dilatation is constant. Therefore $f=h+\alpha \bar{h}$ for some locally univalent analytic function $h$ and a constant $\alpha,|\alpha|<1$. Then $h$ must also map circles to ellipses and by Proposition 1 it is an analytic Möbius transformation.

\section{REFERENCES}

1. M. Chuaqui, P. Duren, and B. Osgood, The Schwarzian derivative for harmonic mappings, J. Analyse Math. 91 (2003), 329-351. MF2037413 (2004j:30030)

2. P. Duren, Harmonic Mappings in the Plane, Cambridge University Press, Cambridge, U.K., 2004. MR2048384

Facultad de Matemáticas, P. Universidad Católica de Chile, Santiago, Chile

E-mail address: mchuaqui@mat.puc.cl

Department of Mathematics, University of Michigan, Ann Arbor, Michigan 481091109

E-mail address: duren@umich.edu

Department of Electrical Engineering, Stanford University, Stanford, California 94305

E-mail address: osgood@stanford.edu 